\title{
Pseudoaneurisma de artéria pediosa: um relato de caso
}

\author{
Pseudoaneurysm of the dorsalis pedis artery: a case report \\ Pseudoaneurisma de la arteria dorsalis pedis: reporte de caso
}

Recebido: 01/12/2021 | Revisado: 06/12/2021 | Aceito: 13/12/2021 | Publicado: 21/12/2021

\author{
Jhonantas Henrique Brito Santos \\ ORCID: https://orcid.org/0000-0001-9790-6041 \\ Universidade Federal do Delta do Parnaíba, Brasil \\ E-mail: jhonantas.brito@gmail.com \\ Breno Vitor Rodrigues Coqueiro Santana \\ ORCID: https://orcid.org/0000-0001-6286-9607 \\ Universidade Federal do Delta do Parnaíba, Brasil \\ E-mail: brenovrcs@gmail.com \\ Camila de Maria Ferreira Brandão \\ ORCID: https://orcid.org/0000-0002-3523-2026 \\ Universidade Federal do Delta do Parnaíba, Brasil \\ E-mail: camilademariafb@gmail.com \\ Cláudio Vinícius Barroso Queirós de Lima \\ ORCID: https://orcid.org/0000-0002-4047-8637 \\ Universidade Federal do Delta do Parnaíba, Brasil \\ E-mail: cld.vinicius99@gmail.com \\ Francisco Nogueira do Rego Neto \\ ORCID: https://orcid.org/0000-0001-6633-1425 \\ Universidade Federal do Delta do Parnaíba, Brasil \\ E-mail: nogueirarego@ufpi.edu.br \\ Heliton José Baquil Araujo \\ ORCID: https://orcid.org/0000-0002-9257-5885 \\ Instituto de Educação Superior do Vale do Parnaíba, Brasil \\ E-mail: heliton_jose@hotmail.com \\ Ian Carlos de Oliveira Andrade \\ ORCID: https://orcid.org/0000-0003-0385-6659 \\ Universidade Federal do Delta do Parnaíba, Brasil \\ E-mail: iancoandrade@gmail.com \\ Olívio Joaquim Fonseca Neto \\ ORCID: https://orcid.org/0000-0002-2695-0077 \\ Universidade Federal do Delta do Parnaíba, Brasil \\ E-mail: oliviofonsecaneto@ hotmail.com \\ Priscylla Frazão Rodrigues \\ ORCID: https://orcid.org/0000-0001-7580-516X \\ Universidade Federal do Delta do Parnaíba, Brasil \\ E-mail: priscyllafrazao1995@gmail.com \\ Rafael Santos Correia \\ ORCID: https://orcid.org/0000-0002-5711-7838 \\ Universidade Federal do Delta do Parnaíba, Brasil \\ E-mail: rafae10094@gmail.com \\ Antonino Neto Coelho Moita \\ ORCID: https://orcid.org/0000-0002-4993-2568 \\ Universidade Federal do Delta do Parnaíba, Brasil \\ E-mail: antoninomoita@ufpi.edu.br \\ Eduardo Silva Rochel \\ ORCID: https://orcid.org/0000-0001-8660-4259 \\ Universidade Federal do Delta do Parnaíba, Brasil \\ E-mail: dr.eduardorochel@gmail.com
}

\begin{abstract}
Resumo
$\mathrm{O}$ artigo tem como objetivo relatar o caso de um paciente do sexo masculino com pseudoaneurisma de artéria pediosa, assim como apresentar o manejo do caso. Paciente, 76 anos, do sexo masculino, com hipertensão arterial controlada, ex-tabagista, foi admitido com queixa de caroço no pé esquerdo e dormência nos dedos. Ao exame físico, apresentava nódulo pulsátil em dorso do pé esquerdo. Os pulsos carotídeos e de membros superiores e inferiores eram normais. Após suspeita de aneurisma verdadeiro, foi solicitado Doppler Arterial de Membro inferior esquerdo (MIE). O ultrassom Doppler revelou dilatação aneurismática em artéria do dorso do pé esquerdo com imagem em yin-yang sugestivo de pseudoaneurisma. Realizou-se aneurismectomia de pediosa, bem como ligadura e secção de arteríolas colaterais ao pseudoaneurisma. Efetuou-se então a revisão da hemostasia e, por fim, a síntese da pele com nylon 4.0.
\end{abstract}


Ao término do ato cirúrgico, a motricidade do pé esquerdo do paciente manteve-se preservada e não foi observada alteração da perfusão dos pododáctilos esquerdos. Após 15 dias do procedimento cirúrgico, retirou-se os pontos e foi realizado o desbridamento das bordas, observando necrose de pequenas bordas de ferida operatória. Paciente encontra-se de alta, evoluindo com edema leve de pé, mas sem outras intercorrências, apresentando boa evolução pósoperatória.

Palavras-chave: Artéria; Pseudoaneurisma; Procedimentos cirúrgicos vasculares.

\begin{abstract}
The article aims to report the case of a male patient with pseudoaneurysm of the dorsalis pedis artery, as well as to present the case management. A 76-year-old male patient with controlled arterial hypertension, a former smoker, was admitted complaining of a lump in the left foot and numbness in the toes. On physical examination, he had a pulsatile nodule on the dorsum of his left foot. The carotid and upper and lower limb pulses were normal. After suspicion of true aneurysm, left lower limb Arterial Doppler (LLL) was requested. Doppler ultrasound revealed aneurysmal dilatation in the dorsum left foot artery with a yin-yang image suggestive of pseudoaneurysm. Pedal aneurysmectomy was performed, as well as ligation and section of arterioles combined with pseudoaneurysm. The hemostasis was reviewed and, finally, the skin was synthesized with 4.0 nylon. At the end of the surgery, the patient's left foot motricity was preserved and there was no change in the perfusion of the left toes. Fifteen days after the surgical procedure, the stitches were removed and debridement of the edges was performed, observing the necrosis of small surgical wound edges. The patient is discharged, evolving with mild foot edema, but without other complications, good postoperative evolution.
\end{abstract}

Keywords: Artery; Pseudoaneurysm; Vascular surgical procedures.

\title{
Resumen
}

El artículo tiene como objetivo reportar el caso de un paciente masculino con pseudoaneurisma de la arteria dorsalis pedis, así como presentar el manejo del caso. Varón de 76 años con hipertensión arterial controlada, exfumador, que ingresa por un bulto en el pie izquierdo y entumecimiento en los dedos. Al examen físico presenta un nódulo pulsátil en el dorso del pie izquierdo. Los pulsos de la carótida y de las extremidades superiores e inferiores eran normales. Ante la sospecha de aneurisma verdadero, se solicitó Doppler Arterial (LLL) de miembro inferior izquierdo. La ecografía Doppler reveló dilatación aneurismática en la arteria dorsal del pie izquierdo con imagen de yin-yang sugestiva de pseudoaneurisma. Se realizó aneurismectomía de pedal, ligadura y corte de arteriolas combinado con pseudoaneurisma. Luego revisamos la hemostasia y, finalmente, se sintetizó la piel con nailon 4.0. Al final de la cirugía, la motricidad del pie izquierdo del paciente se mantuvo y no hubo cambios en la perfusión de los dedos del pie izquierdo. A los 15 días del procedimiento quirúrgico se retiraron los puntos y se realizó el desbridamiento de los bordes, observándose la necrosis de los bordes de la herida quirúrgica de pequeño tamaño. El paciente es dado de alta, evoluciona con edema leve del pie, pero sin otras complicaciones, buena evolución postoperatoria.

Palabras clave: Arteria; Pseudoaneurisma; Procedimientos quirúrgicos vasculares.

\section{Introdução}

Os pseudoaneurismas são eventos patológicos vasculares que caracterizam-se pela dilatação arterial superior a $50 \%$ com relação ao diâmetro normal do vaso, o qual geralmente se desenvolve a partir de uma lesão ou defeito na parede da artéria, dando origem a um hematoma pulsátil que é delimitado por paredes oriundas dos tecidos circunvizinhos (tecido conjuntivo), diferente dos aneurismas verdadeiros, tendo em vista que estes são contidos pelas paredes intactas do próprio vaso (Nogueira et al., 2013; Oliveira et al., 2014; Parikh et al., 2017).

Como fatores de risco mais relevantes para o surgimento dos pseudoaneurismas têm-se o uso de drogas anticoagulantes e antiagregantes plaquetários em terapia tripla, idade avançada, sexo feminino pós-menopausa, diabetes e outras doenças cardiovasculares como hipertensão arterial e doença arterial obstrutiva periférica (Peters et al., 2017). Eles podem acometer uma grande parte das artérias localizadas ao redor do pé ou do tornozelo. Dentre elas, a artéria dorsal do pé ou artéria pediosa pode ser um dos vasos acometidos por essa circunstância patológica cardiovascular, embora seja considerado um sítio incomum (Nolan et al., 2020). As lesões traumáticas e causas iatrogênicas (após procedimentos cirúrgicos, por exemplo) são os fatores desencadeantes mais citados em relatos na literatura (Dahman et al., 2021; Erkut \& Azman, 2019; Parikh et al., 2017; Wiske et al., 2016).

Geralmente, os pseudoaneurismas de artéria pediosa inicialmente manifestam-se de forma indolor, o que até mesmo dificulta a detecção e, consequentemente, atrasa o diagnóstico. No entanto, conforme haja compressão de nervos periféricos 
adjacentes, haverá queixas de dor. Além disso, a percepção de uma massa bem distinta à palpação no dorso do pé, na maior parte das vezes notando-se contratilidade, aliado frequentemente à detecção de sopros auscultáveis, configuram-se como achados clínicos sugestivos para essa condição, orientando-se direcionamento para exames complementares de imagem para confirmação e avaliação da conduta terapêutica (Erkut \& Azman, 2019; Lee et al., 2018; Nogueira et al., 2013; Oliveira et al., 2014; Singh et al., 2013; Yu et al., 2013).

O relato de caso em questão aborda a conduta médica diante de um caso de pseudoaneurisma de artéria pediosa ou artéria dorsal do pé, desde abordagem clínica, diagnóstico, conduta terapêutica adotada e acompanhamento pós-cirúrgico.

\section{Metodologia}

O presente artigo trata-se de um relato de caso, cuja abordagem é descritiva e qualitativa, baseada no método descrito por Pereira et al. (2018). O presente relato visa expor o manejo de paciente com pseudoaneurisma de artéria pediosa, passando pelo manejo clínico e pelo diagnóstico chegando até a conduta terapêutica e o acompanhamento pós-cirúrgico. Para isso, foram reunidos os dados obtidos dos prontuários e dos exames complementares, que foram solicitados para a elaboração do relato. A publicação deste caso foi reconhecida pelo paciente por meio da assinatura do termo de consentimento livre esclarecido (TCLE), de modo que se respalda nos princípios estabelecidos pelo comitê de ética institucional, bem como segue as diretrizes internacionais previstas na declaração de Helsinque.

\section{Relato de Caso}

S.V.S, homem de 76 anos, hipertensão arterial controlada, ex-tabagista, foi admitido com queixa de caroço no pé esquerdo e dormência nos dedos. Negava história de trauma, punção ou cirurgia na região acometida. Ao exame físico, apresentava nódulo pulsátil em dorso do pé esquerdo. Os pulsos carotídeos e de membros superiores e inferiores eram normais. Em primeiro momento, suspeitou-se de aneurisma verdadeiro, sendo então solicitado Doppler Arterial de Membro inferior esquerdo (MIE). O ultrassom Doppler revelou dilatação aneurismática em artéria do dorso do pé esquerdo com imagem em yin-yang sugestivo de pseudoaneurisma. Nas demais artérias, o fluxo trifásico foi satisfatório, não observando nenhum achado patológico (Figura 1). Isto posto, foi solicitado o procedimento cirúrgico. 
Figura 1.

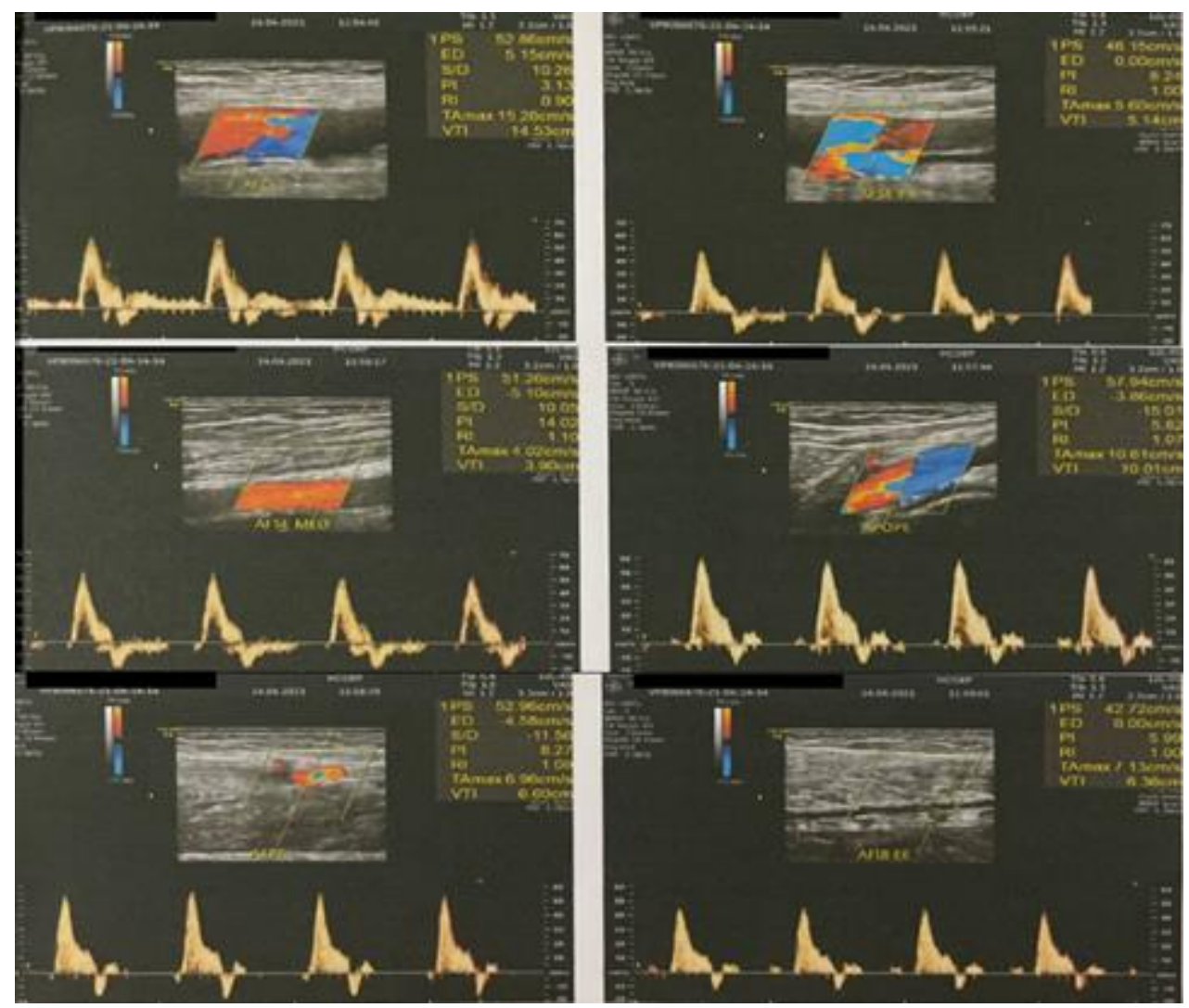

Fonte: Autores.

Realizou-se aneurismectomia de pediosa com dupla ligadura e fio de algodão 2.0 (Figura 2). O procedimento ocorreu na região proximal e distal da artéria pediosa nas regiões pré e pós-aneurisma (Figura 3). Ademais, foi feita ligadura e seç̧ão de arteríolas colaterais ao pseudoaneurisma, além de sua remoção por completo (Figura 4). Efetuou-se então a revisão da hemostasia e, por fim, a síntese da pele com nylon 4.0 (Figura 5). Ao término do ato cirúrgico, bem como no pós-operatório subsequente, a motricidade do pé esquerdo do paciente manteve-se preservada e não se observou alteração da perfusão dos pododáctilos esquerdos, sendo está mantida com enchimento capilar menor que 3 segundos. Após 15 dias do procedimento cirúrgico, retirou-se os pontos e foi realizado o desbridamento das bordas, observando necrose de pequenas bordas de ferida operatória. Paciente encontra-se de alta, evoluindo com edema leve de pé, mas sem outras intercorrências, apresentando boa evolução pós-operatória. 
Research, Society and Development, v. 10, n. 17, e69101724157, 2021

(CC BY 4.0) | ISSN 2525-3409 | DOI: http://dx.doi.org/10.33448/rsd-v10i17.24157

\section{Figura 2.}

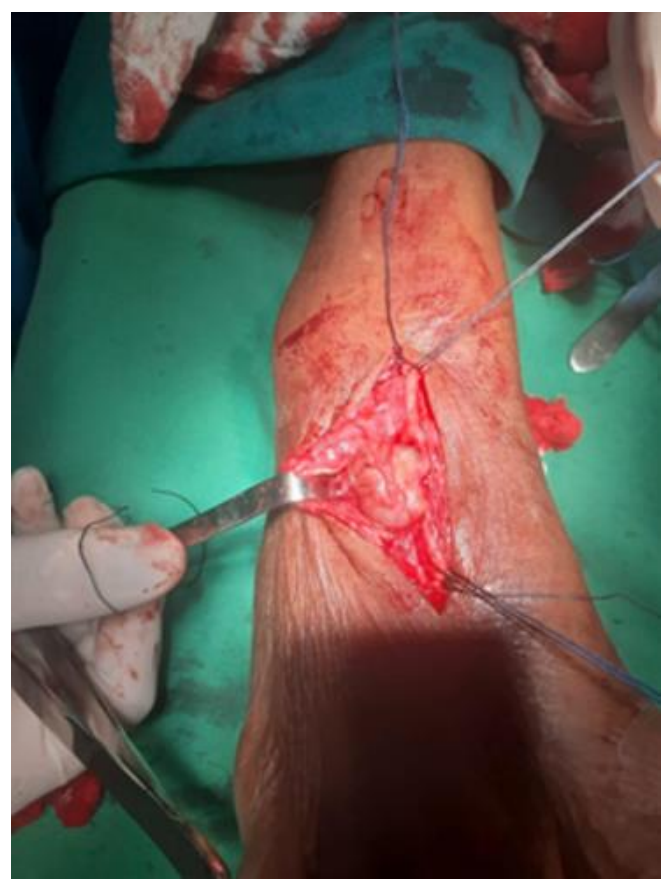

Fonte: Autores.

Figura 3.

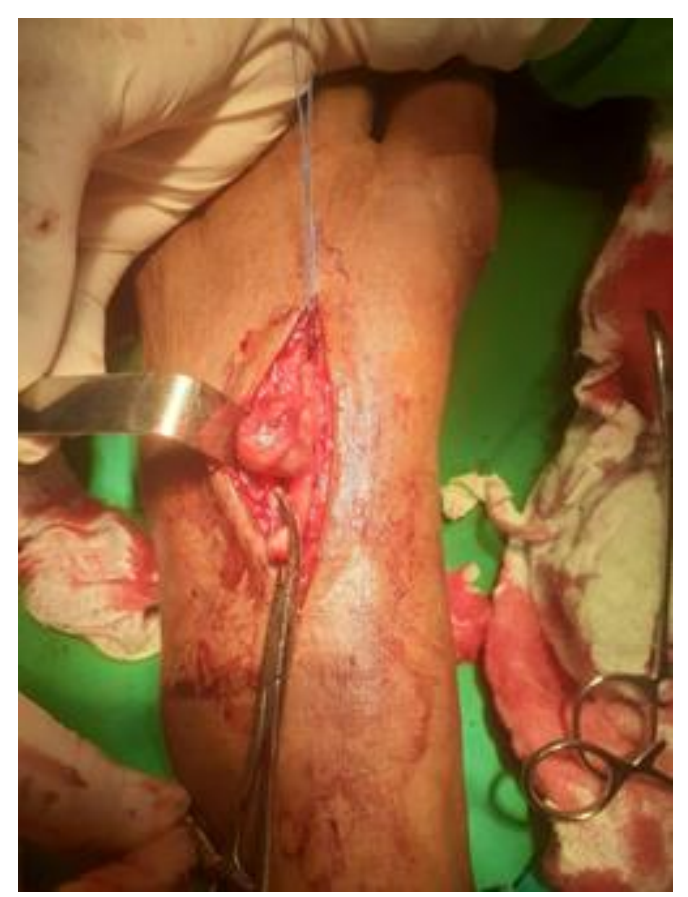

Fonte: Autores. 
Figura 4.

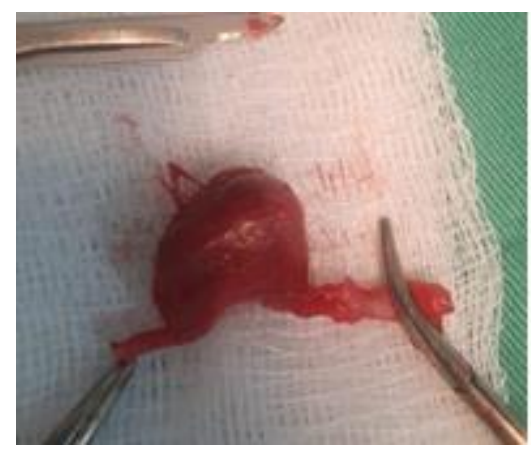

Fonte: Autores.
Figura 5.

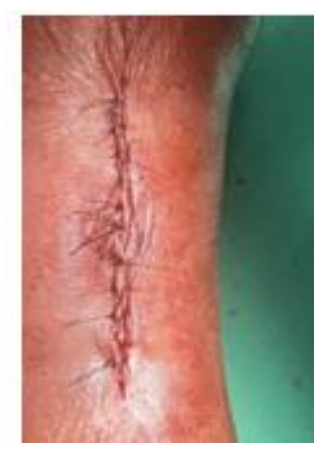

Fonte: Autores.

\section{Discussão}

Os pseudoaneurismas se diferenciam dos aneurismas verdadeiros pela forma que cada um acomete a parede vascular. $\mathrm{O}$ verdadeiro se caracteriza por ter o envolvimento das três camadas que compõem o vaso: íntima, média e adventícia, enquanto no "falso" aneurisma, há lesão dessas camadas, levando a extravasamento de sangue (Miyamotto et al., 2004), sendo ele contido pelos tecidos que circundam a artéria, (Oliveira et al., 2014).

A ocorrência de pseudoaneurismas no pé, mais especificamente na artéria dorsal do pé, é uma complicação rara, com poucos relatos na literatura, embora exista a crença de que a prevalência real seja significantemente maior que a relatada (McKee et al., 2000). Sua etiologia é geralmente associada a sequelas de eventos que lesam a parede arterial: traumas, como a ocorrência de entorses no tornozelo e de lacerações; cirurgias, a exemplo de artroscopias e de fixação de fratura e infecções. Outros predisponentes que valem destaque são: doença de Marfan, síndrome de Ehlers-Danlos, doença de Behcet, displasia fibromuscular tipo IV e osteogênese imperfeita, além de diabetes, desnutrição e imunossupressão (Bausells et al., 2003; Grzela et al., 2019; Yu et al., 2013).

No entanto, existem ainda escassos estudos que relatam causas idiopáticas, como é o caso do presente relato, em que o paciente nega a ocorrência de qualquer um desses eventos. Apesar disso, é importante que a hipótese de trauma seja pesquisada mais profundamente, uma vez que o aneurisma nem sempre se manifesta logo após o episódio agudo, mesmo que isso seja o mais comum, podendo aparecer até 5 anos após o evento traumático (Yu et al., 2013).

Ao se manifestar, o diagnóstico do pseudoaneurisma pode ser feito clinicamente, por meio da palpação de um hematoma pulsátil, raramente associado a dor, exceto em casos de tamanho aumentado ou de compressão de algum nervo periférico (Yu et al., 2013). Entretanto, a confirmação do diagnóstico por meio de exames complementares de imagem também possui papel importante (Oliveira et al., 2014). Ainda, há estudos que instruem, no caso de aneurisma nesse local, que haja uma pesquisa de outras ocorrências por meio de um meticuloso exame clínico e exames de imagem (Dahman et al., 2021; Fitzpatrick, 1980).

Para essa confirmação, a ultrassonografia Doppler se apresenta como melhor opção, por não ser invasiva e não requerer o uso de agentes de contraste. Além disso, esse método apresenta alta sensibilidade (94\%) e alta especificidade (97\%) para diagnóstico de pseudoaneurismas, portanto, sendo ideal para distinguir essas afecções de outras massas pulsáteis (Coughlin \& Paushter, 1988; Mahmoud et al., 2015; Özdemir et al., 2003).

No caso em questão, a apresentação do padrão "yin-yang" no resultado do exame altera o diagnóstico para o pseudoaneurisma, em contradição à hipótese de aneurisma verdadeiro levantada inicialmente. O motivo disso é que, durante a sístole, o fluxo anterógrado passa para o pseudoaneurisma pela falha e, durante a diástole, o aumento de sua pressão em relação à da artéria subjacente resulta em fluxo retrógrado para fora, pela falha, e de volta para a artéria. Isso se manifesta em um padrão Doppler pulsado "para frente e para trás" característico, no colo do pseudoaneurisma, e produz um padrão de fluxo 
colorido em redemoinho "yin-yang" (Mahmoud et al., 2015; Özdemir et al., 2003).

Dessa forma, a ultrassonografia com Doppler estabeleceu o diagnóstico de certeza e foi suficiente para definir o planejamento cirúrgico a ser adotado, visto que forneceu a localização exata da lesão e a sua relação com as estruturas vizinhas, bem como confirmar a integridade das demais artérias e ausência de outras alterações vasculares.

Existem diferentes formas de tratamento para o pseudoaneurisma de artéria dorsal do pé, tais como ressecção e ligadura simples, revascularizações com anastomose término-terminal ou interposição de veia safena (Ferreira et al., 2018; Maydew, 2007; Vlachovsky et al., 2017). Contudo, no caso discutido, o cirurgião optou por realizar uma aneurismectomia de pediosa com dupla ligadura, também a checagem da motricidade e da perfusão do pé após o procedimento cirúrgico.

Por fim, os exames pré-operatórios, o anatomopatológico e a investigação clínica, não evidenciaram a etiologia da doença.

\section{Conclusão}

Portanto, apesar do "falso" aneurisma podálico ser categorizado como uma complicação rara, as condutas de prevenção, no que tange a essa doença, não devem ser menosprezadas ou ignoradas. Vale lembrar que existem alguns fatores predeterminantes para o desenvolvimento do quadro, tanto genéticos, em se tratando da Síndrome de Marfan, quanto físicos, estabelecendo-se uma relação direta entre um trauma local e a manifestação tardia dos sinais e sintomas. Um acompanhamento médico, frequente e eficaz, é de suma importância para evitar o desenvolvimento do pseudoaneurisma.

O diagnóstico precoce se mostra essencial para um bom prognóstico da doença. O exame de Ultrassonografia Doppler foi evidenciado como o melhor teste diagnóstico para a detecção de um aneurisma, disponibilizando informações necessárias para uma boa conduta terapêutica e um bom planejamento cirúrgico. Atualmente, as diferentes condutas adotadas para um melhor manejo do paciente favorecem a resolução de vários quadros com diferentes manifestações singulares. O paciente abordado nesse relato recebeu um tratamento adequado e um bom acompanhamento pós-operatório, progredindo com uma melhora substancial de sua condição primária.

Por fim, com o intuito de compreender melhor sobre a fisiopatologia do pseudoaneurisma e ofertar condutas terapêuticas mais elaboradas, as pesquisas voltadas para o pseudoaneurisma devem ser constantemente incentivadas. Desse modo, as equipes de saúde especializadas estarão ainda mais aptas para proporcionar um desfecho ideal de tratamento. Como proposta para execução de trabalhos científicos futuros, sugere-se uma pesquisa que melhor aborde a eficácia entre as opções terapêuticas disponíveis para o tratamento dos pseudoaneurismas das artérias da região dos pés.

\section{Referências}

Bausells, M. I., Casagrande, M. R., Miquelin, D. G., \& Reis, L. F. (2003). Aneurisma da artéria dorsal do pé: Relato de caso e revisão da literatura. Jornal Vascular Brasileiro, 2(1), 26-28.

Coughlin, B. F., \& Paushter, D. M. (1988). Peripheral pseudoaneurysms: Evaluation with duplex US. Radiology, 168(2), 339-342. https://doi.org/10.1148/radiology.168.2.3293107.

Dahman, M., Alhomaidi, Y., Albattah, M., Howil, A. A., Alsultan, A. M., \& Alenazi, N. (2021). Dorsalis pedis artery pseudoaneurysm: A rare entity in the field of vascular surgery. International journal of surgery case reports, 86, 106302. https://doi.org/10.1016/j.ijscr.2021.106302.

Erkut, B., \& Ates, A. (2019). Post-Traumatic Dorsalis Pedis Pseudo-aneurysm Caused by Crush Injury. EJVES short reports, 44, 29-32. https://doi.org/10.1016/j.ejvssr.2019.07.003.

Ferreira, U. G., Aragão, J. A., Lenik, A. A., Aragão, I. C. S., Aragão, F. M. S., Leão, W. B., Nunes, C. E., \& Reis, F. P. (2018). Aneurisma verdadeiro de artéria dorsal do pé: Relato de caso. Jornal Vascular Brasileiro, 17(2), 152-155. https://doi.org/10.1590/16737-5449.012817.

Fitzpatrick W. H. (1980). Idiopathic aneurysm of the dorsalis pedis artery. The Journal of foot surgery, 19(4), 185-186.

Grzela, T., Piotrowicz, R., \& Leksowski, K. (2019). Should the Histological Examination Be Used in the Diagnosis of Aneurysms of the Dorsalis Pedis Artery? A Case Report and Review of the Literature. Annals of vascular surgery, 54, 336.e1-336.e4. https://doi.org/10.1016/j.avsg.2018.06.023. 
Lee, Y., Ryu, H. Y., Kim, Y. J., \& Ku, G. W. (2018). Unusual Pseudoaneurysm of the Dorsalis Pedis Artery after an Iatrogenic Injury. The Korean journal of thoracic and cardiovascular surgery, 51(3), 213-215. https://doi.org/10.5090/kjtcs.2018.51.3.213.

Mahmoud, M. Z., Al-Saadi, M., Abuderman, A., Alzimami, K. S., Alkhorayef, M., Almagli, B., \& Sulieman, A. (2015). "To-and-fro" waveform in the diagnosis of arterial pseudoaneurysms. World journal of radiology, 7(5), 89-99. https://doi.org/10.4329/wjr.v7.i5.89.

Maydew, M. S. (2007). Dorsalis pedis aneurysm: ultrasound diagnosis. Emergency Radiology, 13(5), 277-280.

McKee, T. I., \& Fisher, J. B. (2000). Dorsalis pedis artery aneurysm: Case report and literature review. Journal of Vascular Surgery, 31(3), 589-591. https://doi.org/10.1067/mva.2000.102130.

Miyamotto, M., Moreira, R. C. R., Erzinger, F. L., \& França, G. J. (2004). Pseudo-aneurisma idiopático da artéria poplítea. Jornal Vascular Brasileiro, 3(2), $169-171$.

Nogueira, A. C. S., Salgado, C. G., Nogueira, F. B. S., Amaral, S. I., \& Rabischoffsky, A. (2013). Pseudoaneurismas: Quando e Como Tratá-Los. Arquivos Brasileiros de Cardiologia: imagem cardiovascular, 26(4), 289-307.

Nolan, F. C., Bourke, M., Kenny, A., \& Moloney, T. (2020). Dorsalis pedis artery aneurysm. BMJ Case Reports, 13(2), e231969. https://doi.org/10.1136/bcr2019-231969.

Oliveira, G. P., Guillaumon, A. T., Brito, I. B. de, Lima, J. M. T., Benvindo, S. C., \& Santos, L. G. dos. (2014). Idiopathic popliteal artery pseudoaneurysm: Emergency diagnosis and treatment. Jornal Vascular Brasileiro, 13(3), 244-248. https://doi.org/10.1590/jvb.2014.032.

Özdemir, H., Mahmutyazıcıŏ̆lu, K., Özkökeli, M., Savranlar, A., Özer, T., \& Demirel, F. (2003). Pseudoaneurysm of the dorsalis pedis artery: Color Doppler sonographic and angiographic findings: Pseudoaneurysm of the Dorsalis Pedis Artery. Journal of Clinical Ultrasound, 31(5), $283-287$. https://doi.org/10.1002/jcu.10164.

Parikh, S., Dawe, E., Lee, C., Whitehead-Clarke, T., Smith, C., \& Bendall, S. (2017). A cadaveric study showing the anatomical variations in the branches of the dorsalis pedis artery at the level of the ankle joint and its clinical implication in ankle arthroscopy. Annals of the Royal College of Surgeons of England, 99(4), 286-288. https://doi.org/10.1308/rcsann.2016.0288.

Pereira, A. S., Shitsuka, D. M., Parreira, F. J., \& Shitsuka, R. (2018). Metodologia da pesquisa científica. UFSM.

Peters, S.; Braun-Dullaeus, R.; Herold, J. Pseudoaneurysm: Incidence, Therapy and Complications. Hämostaseologie, v. 38 (03), 166-172, 2017.

Singh, S., Arora, S., Thora, A., Mohan, R., Sural, S., \& Dhal, A. (2013). Pseudoaneurysm of profunda femoris artery following dynamic hip screw fixation for intertrochanteric femoral fracture. Chinese journal of traumatology = Zhonghua chuang shang za zhi, 16(4), 233-236.

Vlachovsky, R., Staffa, R., \& Novotny, T. (2017). Pseudoaneurysm of the dorsalis pedis artery: case report and literature review. The Journal of Foot and Ankle Surgery, 56(2), 398-400.

Wiske, C. P., Itoga, N. K., Ullery, B. W., Hunt, K. J., \& Chandra, V. (2016). Ruptured Pseudoaneurysm of the Dorsalis Pedis Artery Following Ankle Arthroscopy: A Case Report. JBJS case connector, 6(4), e102. https://doi.org/10.2106/JBJS.CC.16.00069.

Yu, J. L., Ho, E., \& Wines, A. P. (2013). Pseudoaneurysms around the foot and ankle: Case report and literature review. Foot and Ankle Surgery, 19(3), 194198. https://doi.org/10.1016/j.fas.2012.08.003. 